\title{
TERAPEUTICA Erradicar el Helicobacter pylori antes de tratar con antiinflamatorios disminuye las úlceras gastroduodenales
}

Randomised trial of eradication of Helicobacter pylori before non-steroidal anti-inflamatory drug therapy to prevent peptic ulcers. Chan FKL, Sung JJY, Sydney Chung SC y col. Lancet. $1997 ; 350: 975-79$

\section{Objetivo}

Evaluar si la erradicación del Helicobacter pylori (HP) antes del inicio de un tratamiento prolongado con anti-inflamatorios no esteroides (AINE) podría prevenir la aparición de úlceras gastroduodenales

\section{Diseño}

Ensayo clínico randomizado.

Lugar

Hong Kong. Práctica ambulatoria.

\section{Pacientes}

Se incluyeron 92 pacientes que cumplían con los siguientes criterios: 1. Trastornos musculoesqueléticos con indicación de AINE prolongado; 2. HP por endoscopía; 3 . Sin antecedentes de úlcera ni de exposición a AINE. Edad promedio 61 años, el $63 \%$ presentaba al menos una comorbilidad seria. pos recibieron naproxeno $750 \mathrm{mg} /$ día durante 8 semanas; pero en el grupo de la intervención (45 pacientes), previo al inicio del naproxeno, se hizo un curso de una semana con subcitrato de bismuto $120 \mathrm{mg}$, tetraciclina $500 \mathrm{mg}$ y metronidazol $400 \mathrm{mg}$ (todo 4 veces por día), con la intención de erradicar el HP.

\section{Medición de resultados principales}

Incidencia de úlceras gastroduodenales en la endoscopía luego de 8 semanas de tratamiento con naproxeno. En este procedimiento se confirmó también la erradicación o no del HP.

\section{Resultados principales}

Presentaron úlcera el 7\% (n=3) de los pacientes tratados y el $26 \%(\mathrm{n}=12)$ del grupo control $(\mathrm{p}=0.01)$. Se erradicó exitosamente el HP en el $89 \%(n=40)$ del grupo tratado y en ninguno del grupo control. En dos de los tres pacientes que desarrollaron úlcera en el grupo tratado, no se había logrado erradicar el HP.

\section{Conclusiones}

La erradicación del HP, antes del inicio del tratamiento con AINE, redujo la aparición de úlceras gastroduodenales.

Fuente de financiamiento: No referida

\section{Intervención}

A los 92 pacientes se los randomizó en dos grupos. Ambos gru-

\section{Comentario}

La prevención de las úlceras inducidas por AINE y sus complicaciones sigue siendo un problema sin solución. La ranitidina y el sucralfato no demostraron eficacia en la prevención, sobre todo en lo referente a úlceras gástricas. El omeprazol y la famotidina (1) han demostrado cierta utilidad y sólo el misoprostol ha dado resultados consistentes (2). Desgraciadamente, su costo y frecuentes efectos adversos (diarrea) impiden hacer una recomendación generalizada de su uso en cualquier paciente con indicación crónica de AINE. Este trabajo, por su efectividad y bajo costo, plantea una posibilidad muy interesante. Sin embargo, deben hacerse ciertas consideraciones. En primer lugar, este es uno de los primeros estudios que sugiere una relación entre HP y úlcera péptica provocada por AINE, lo que no pudieron demostrar estudios previos (34). Por otro lado, hay que considerar que el resultado medido en este estudio fue la reducción de las úlceras detectadas por endoscopía. Es sabido que el tratamiento prolongado con AINE provoca frecuentemente úlceras gastroduodenales; sin embargo, gran parte de estas úlceras son asintomáticas y no ocasionan complicaciones. El principal objetivo de un tratamiento profiláctico de la enfermedad ulcerosa es reducir la incidencia de síntomas y complicaciones (sangrado y perforación). Si bien es muy probable que ésto se logre mediante un tratamiento que prevenga la aparición de lesiones endoscópicas, habría que esperar un ensayo clínico con mayor número de pacientes para confirmar esta hipótesis. Por último, en este estudio se incluyeron pacientes de edad avanzada con importante comorbilidad, grupo de alto riesgo para úlceras gastroduodenales asociadas a AINE. Por lo tanto, se debe ser cauto al extender las conclusiones de este estudio a otras poblaciones. Lo que hoy podemos hacer es evitar, cuando sea posible, los tratamientos prolongados con AINE, intentar tratamientos locales o utilizar paracetamol. Cuando sea necesario, usar preferentemente AINE de menor toxicidad gastrointestinal (Ibuprofeno) y evitar los de mayor toxicidad (piroxicam, ketoprofeno) (5). En los pacientes de riesgo (mayores de 65 años, con comorbilidad -en especial cardiovascular-, antecedentes de enfermedad ulcerosa o pacientes que van a recibir altas dosis) podemos considerar una medida profiláctica. Idealmente misoprostol (200 mg. 4 veces por día) o, en caso de intolerancia, omeprazol o famotidina. En el futuro, el desarrollo de AINE inhibidores selectivos de la ciclo-oxigenasa II como el nabumetona ( $\sin$ toxicidad gastroduodenal) quizás aporten una solución definitiva. En cuanto a la erradicación del HP, habrá que esperar otros estu- dios que confirmen esta hipótesis y, sobre todo, poder interpretar estos resultados en un contexto donde el rol del HP en todos los aspectos clínicos se encuentre más aclarado que en la actualidad.

Dr. Andrés Pichon Rivière Sistemas de Protección Médica

\section{Referencias}

1. Táha AS, Hudson N, Hawkey CJ, et al. Famotidine for the prevention of gastric and duodenal ulcers caused by nonsteroidal antiinflamatory drugs. N Eng J Med 1996; 334: 1435-39

2. Stucki G, Johannesson M, Liang M. Is Misoprostol Cost-effective in the Prevention of Nonsteroidal Anti-inflammatory Drug-induced Gastropathy in Patients With Chronic arthritis? Arch In Med 1994; 154:2020-25

3. Schubert T, Bologna S, Nensey Y, Schubert A, Mascha E. Ulcer risck factors: Interactions between Helicobacter pylori infection, Nonsteroidal use, and age. Am J Med 1993; 94:41318.

4. Roth S. NSAID Gastropathy. Arch In Med 1996: 156: 1623-28.

5. Langman MJS, Wainwright P, Lawson DH, Rawlins MD, Logan RFA, Vessey MP, Colin-Jones DG. Risks of bleeding peptic ulcer associated with individual non-steroidal anti-inflammatory drugs. Lancet 1994; 343: 1075-78. 